\title{
OMICS as Therapeutic Platform: Environmental Factors to Parkinson's Disease
}

\author{
Hong Duck Kim ${ }^{1 *}$, Shana Warner ${ }^{2}$, Kamini Singh ${ }^{1}$, Jae-Hyeon Cho ${ }^{3}$ and Diane E Heck ${ }^{1}$ \\ ${ }^{1}$ Department of Environmental Health Science, School of Health Sciences and Practice, New York Medical College, Valhalla, NY 10595, USA \\ ${ }^{2}$ Department of Epidemiology, School of Health Sciences and Practice, New York Medical College, Valhalla, NY 10595, USA \\ ${ }^{3}$ Institute of Agriculture and Life Science, College of Veterinary Medicine, Gyeongsang National University, Jinju 660-701, Korea
}

Keywords: Parkinson disease; Industrialization; Mutations; Neurodegenerative disease

\section{Background}

Environmental pollutants and toxicants take many forms as they can be chemical, biological and physical in nature. Urbanization and industrialization have led to increasingly high amounts of waste production and discharge into the environment. Everyday living places people in constant contact with environmental toxicants through contaminated mediums of air, water and land. The health implications suffered include much of which is encountered through air pollution. Respiratory ailments such as asthma allergies, and eye and nasal passage irritation are common symptoms of exposure. The immune, endocrine and reproductive systems are susceptible to harm as well. It has been documented that exposure to particulates are associated with morbidity and mortality risks from many diseases, chronic illnesses and even cancer [1]. Increasing lines of evidence support that neurodegenerative disease such as Parkinson disease (PD) may be associated with multiple etiological components including genetic factors (i.e., NFE2L2, SNCA, LRRK2, Parkin, PINK, DJ-1, and ATP13A2) [2-6], and environmental factors (i.e., smoking, aging, pesticide, herbicide, infections, or various environmental chemicals) [7-10].

The mitochondria are special organelles located within cells that contain their own DNA as well as important organelle to maintain cell homeostasis reflect responsible for biochemical processes of respiration and energy production. They are essentially the power house of the cell by producing ATP as a driving fuel for the cells [11]. Interestingly, the mitochondria are the focal point for idiopathic diseases and genetic diseases due to their sensitivity to environmental insult. In previous, scientific reports are making connections between mitochondrial dysfunction include mutation and resulting health conditions such as Parkinson disease (PD), Alzheimer disease (AD), and autism and even cardiovascular illness [12-15]. Perier and Vila [16] have described that mitochondria have important cellular functions that include oxidative phosphorylation, regulation of calcium homeostasis, lysosomal impairment, and control of program cell death. Although the mitochondria are equipped for such complex roles, they seem to have susceptibilities to mutations from environmental factors that can lead to certain human diseases.

It remains unclear how environmental factors and genetic disposition may attribute to development of idiopathic neurodegenerative disorders. It is noteworthy, that there is evidence to support PD etiology due to mitochondria dysfunction. This could be linked idiopathically to PD pathogenesis through alteration of functional molecular mechanisms such as protein Parkin, $\alpha$-Synuclein, aberrant genetic modification such as mutant PARK2 and parkin mutations in the patient samples [17-22].

Parkinson's disease (PD) is a neurodegenerative disease that slowly causes dopaminergic neurons in the midbrain to die. This gradual loss of dopamine causes loss of coordinating muscle movements. Perier and
Villa [16] have also stated that "defective mitochondrial respiration has long been associated with the pathogenesis of PD". The evidence for this claim came about when drug abusers were exposed to 1-methly4-phenyl-1,2,3,4-tetrahydropyridine (MPTP). MPTP selectively kills dopaminergic neurons of the susbstania nigra pars compacta (SNpc) [16]. In previous studies, it has been shown that blockade of Complex I has reduced Complex I activity in the brain and skeletal muscles of those with Parkinson's disease. Other alterations of the mitochondria that can cause this neurodegenerative disease includes increased production of mitochondria reactive oxygen species (ROS), ROS mediated mitochondria DNA (mtDNA) damage, bioenergetics failure, and activation of mitochondrial apoptotic pathways [16]. All of this evidence supports the premise that dysfunctions in the dynamic mitochondria can definitely cause Parkinson's symptoms to develop in organisms. Just like reports of mutations in the mitochondria the environment can also alter the mitochondria and increase the risk of Parkinson's disease [22,23].

In accordance with previous reports, it has also been shown that exposure to certain environmental factors can cause malfunctions in the mitochondria leading to certain human diseases. The combined effect of environmental influences and mitochondrial susceptibilities may be contributing factors to Parkinson's disease. According to National Institute of Environmental Health Science (NIEHS, 2016), "pesticide exposure is associated with an increased risk of developing Parkinson's disease". Those who come in to contact with pesticides are more prone to develop Parkinson's disease. Occupational use of two specific of pesticides, rotenone and paraquat poisoning, are documented to be associated with PD [24] "Rotenone directly inhibits the function of the mitochondria, while paraquat increases the production of certain damaging oxygen derivatives" (NIEHS, 2016). This highlights the cellular consequence of mitochondrial malfunction in individuals with specific pesticide exposure, especially rotenone and paraquat, and the subsequent increased risk of developing neurodegenerative disease.

Parkinson's disease (PD) is still undergoing tremendous research to understand how genes, environment, and mitochondria all interact. Currently there are no standard diagnostic tests for PD, however neurological history and physical examination do determine the presence of disease in an individual. Although there is no cure for PD, many

*Corresponding author: Hong Duck Kim, Department of Environmental Health Science, School of Health Sciences and Practice, New York Medical College, Valhalla, NY 10595, USA, Tel: 914-594-4259; E-mail: hongduck_kim@nymc.edu

Received May 13, 2016; Accepted June 07, 2016; Published June 14, 2016

Citation: Kim HD, Warner S, Singh K, Cho JH, Heck DE (2016) OMICS as Therapeutic Platform: Environmental Factors to Parkinson's Disease. J Microb Biochem Technol 8: 222-225. doi: 10.4172/1948-5948.1000289

Copyright: ( $2016 \mathrm{Kim} \mathrm{HD}$, et al. This is an open-access article distributed under the terms of the Creative Commons Attribution License, which permits unrestricted use, distribution, and reproduction in any medium, provided the original author and source are credited. 
treatments exist to help minimize the symptoms. Carbidopa-levodopa is the most effective treatment in which the chemical passes through the brain and converts into dopamine [25]. Conjugated Dopamine or combination pharmacological agents with cysteine can help stabilize the effects of PD by preventing the oxidation process of dopamine to dopamine o-quinone, aminochrome and 5,6-indolequinone. This treatment seems to be effective against disease progression [26,27]. Unfortunately long term treatment with levodopa (L-DOPA) will cause tardive dyskinesia in addition to having a malfunctioning nigrostriatal dopaminergic system $[28,29]$. Another treatment involves the use of is MAO-B inhibitors. There are several reports documenting that these inhibitors could prevent the breakdown of dopamine by inhibiting the brain enzyme monoamine oxidase (MAO-B) [30-32]. This drug can prevent metabolism of dopamine. Parkinson's disease (PD) is a difficult neurodegenerative disease to control. Treatments are limited to delaying disease progression and treating the symptoms. Utilizing advanced technologies can lead to invaluable insight of rapid disease progression and what biological processes need to be targeted in order to prevent $\mathrm{PD}$ from occurring.

\section{Problem Statement}

Of particular interest is Parkinson's disease. The exact etiology remains unclear but a common thread of thought it that there are combined causal roots of genetics and environment. Approximately $5-10 \%$ cases are known to be due to genetic causes (monogenic form) in PD [33,34]. Environmental risks are from a number of sources that include most commonly pesticide exposure, living in rural settings, well water consumption, herbicide exposure and living in proximity to industrial plants or quarries. Bellou et al. reported that environmental factors such as dietary factors, drugs, medical history or comorbid diseases, exposure to toxic environmental agents and habits have been substantially associated with Parkinson's disease in 21 of 75 metaanalyses (more than 1000 cases) [35].

Parkinson's disease affects $1 \%$ of people over the age of 60 . The incidence is 5 to 24 cases per 100,000 and prevalence is anywhere from 66 to 187 cases per 100,000 . Most studies yield a prevalence of 120 cases per 100,000 . Both disease incidence and prevalence increase with age; the average age of onset is 60 years old. Early onset of disease under the age of 40 is uncommon but possible. It is also 1.5 times more likely to occur in men than in women $[36,37]$.

Mitochondria malfunction can be linked to certain neurological disorders which might be considered as a significant role in the pathogenesis of PD. Those with susceptible genes are more prone to developing the disease and experiencing rapid progression if exposed to certain environmental factors.

\section{Methodology}

Since the advent of enhanced technologies, scientists have been able to use high sequencing techniques (i.e., next gene sequencing, NGS and RNA sequencing), microarrays, and many other technologies such as gene editing technology to analyze the function of the body at molecular levels, known as OMICS [38,39]. OMICS is broken down into four major categories: Genomics is the study of the genes; transcriptomics is the study of the mRNA; proteomics is the study of functional proteins, and metabolomics is the study of cellular metabolism. The use of OMICS has allowed the biomedical community to analyze the effects of the environment at the molecular and cellular levels in organisms in order to understand how the biological processes can be affected. This can lead to better risk assessments, accurate diagnoses of diseases, and the practice of personalized medicine in the future.

Risk assessment is the measurement of adverse health effects in a population that is exposed to risk enhancing factors such as environmental contaminants. According to the Environmental Protection Agency (EPA) guidance, the four steps in risk assessment are described as the following: 1. Identify the hazard, 2. Examine the doseresponse relationship, 3. Exposure assessment and 4. Characterization risk [40]. However, current research shows a lack of OMICS usage in risk assessment. The EPA (2013) has reported that OMICS studies are lacking dose-response and don't know which exposure dose causes an appearance of the first adverse effects. This may emphasize that OMICS technologies are lacking in risk assessment but other areas show great promise.

Utilizing personalized medicine allows for risk evaluation, diagnosis, monitoring, and treatment of patients according to their specific genetic and molecular makeup. Chen et al. [41] has reported that "the disease risk can be evaluated from genome sequencing and shows how traits associated with diseases can be monitored to identify varying physiological stages". They also stated that "detailed OMICS profiling with genomic sequencing can provide molecular and physiological information of medical significance". Since the mitochondria are known for its dynamic nature, it can be mutated from environmental factors leading to certain neurological diseases. Genomics can be useful to identify biomarkers in people who suffer from Parkinson's disease to assess risk, monitor prognosis, determine treatments, and possible preventions in the future.

\section{Outcomes}

Advances in OMICS techniques have aided scientists to discover cellular mechanisms in how the physiological and molecular state changes in organisms with diseases. These advance technologies have allowed for identification of biomarkers that "can provide a window into disease mechanisms with hope of developing specific therapeutic targets of the disease", as a stated by Caudle et al. [42]. Discovery of biomarkers will help scientist to know how the body functions in normal and disease states with hopes of formulating medicines. Their study also commented that, "OMICS techniques have been integral in confirming pathways that are associated with $\mathrm{PD}$, including mitochondrial and proteasomes function and synaptic neurotransmission" [42]. With the advances in transcriptomics, proteomics, and metabolomics, it is possible to understand how the mitochondria function and relate to the development of PD.

Transcriptomics is the study of mRNA. As it relates to Parkinson's Disease (PD), "disruption of the mitochondria's function can have severe repercussions for the normal functioning of the dopamine neuron, through the suppression of energy production and generation of neurotoxic reactive oxygen species" [42]. This may lead to symptoms of neurodegenerative disease such as those seen in Alzheimer disease (AD), Huntington Disease (HD), and Parkinson Disease (PD). In support of this premise, Caudle et al. [43] has stated that "exposure of rats and mice to mitochondrial complex I inhibitors, rotenone and MPTP, causes considerable degeneration of dopamine neurons in SNpc, showing pathological features of PD”. Microarray analysis is a great technique used in analyzing mRNA expression that it can predict gene profile using clinical samples such as urine, blood, cerebrospinal fluid (CSF), and saliva. This technique has helped scientists discover certain biomarkers that are under-regulated in susceptible individuals who are prone to getting PD. Caudle et al. [42] has suggested that "microarray 
analysis and blood from PD patients and controls have uncovered a significant clue in in the transcriptional modulation of $\alpha$-synuclein: genetic alteration of $\alpha$-synuclein can have deleterious effects on the risks of PD". Understanding the mechanism of $\alpha$-synuclein can assist with the invention of therapeutic medicines that suppress deregulation of $\alpha$-synuclein and hopefully control the progression of PD.

Proteomics is one of the most powerful technologies in the field of clinical research. It allows scientists to understand how proteins function in complex biological systems and how possible protein alterations underlie pathological processes. According to Caudle et al. [42] "the oxidation of DJ-1 could have significant consequences on its ability to function in attenuating the generation of oxidative stress within the dopamine neuron and $\mathrm{Cu} / \mathrm{ZN}$ superoxide dismutase (SOD) serves to reduce the amount of intracellular oxidative stress, which can readily interact with other radicals to generate highly toxic reactive species". Another discovery of a specific heat shock protein that can cause PD and mitochondrial repression is mortalin. It has been reported that "mortalin (also known as HSP70 or GRP75) expression was found to significantly reduce dopamine neurons in SNpc of PD patients compared with controls and is specific to mitochondrial fraction when biochemically isolated" [42].

From these findings, it was demonstrated that 72 other proteins have also been known to be altered in PD patients. Identification of these protein alterations can serve as biomarkers in understanding the mechanisms of cellular pathways providing future targeted treatments at mutated or altered proteins in transgenic animals or patients who suffer from PD. So far, proteomics and transcriptomics have uncovered a vast amount of credible scientific information regarding the intricacies of cellular function and the relationship between mitochondria and Parkinson's.

Applying metabolomics can further advance the understanding of PD and mitochondria function. Metabolomics is the study of metabolism in the cellular pathways of organisms. It has been indicated that "reduction in uric acid and increase levels of glutathione and 8-hydroxy-2'-deoxyguanosine have contributed to the role of oxidative stress in PD" [42]. This suggests that PD patients display various levels of certain metabolites throughout the body that can disrupt oxidation leading to progression of PD. By knowing how these levels vary, researchers can create pharmacological remedies aimed at returning the levels to normal and perhaps completely preventing disease occurrence. With the application of OMICS, biomedical science has expanded the molecular understanding of mRNA, proteins, and metabolites and how their disturbances affects the mitochondrial function, leading to PD. Realizing this molecular network connection in the pathogenesis of PD would be beneficial to improve the risk assessment of the environmental factors for this disease.

\section{Potential Impacts}

Research has shown that pesticides can cause mitochondrial disruption that can lead to the development of neurodegenerative diseases such as Alzheimer's and Parkinson's diseases [13]. OMICS technologies have gathered significant data showing how disruption in genes, proteins, metabolites, and cellular pathways affect mitochondrial function that the subsequent decrease in dopamine neurons. Thorough understanding of how Parkinson's can develop has the potential to improve risk assessment and diagnosis. This would greatly contribute to the preservation of public health.

Some public health implications would be to resolve the issue by using alternative pesticides that contain safer environmental compounds. Pesticides contain a lot of hazardous substances and chemicals that can cause disruption in the cellular pathways. Pollutants or different toxicants can readily accumulate in mitochondrial membranes due to its high lipid content as seen with the antineoplastic agent, adriamycin. The lipid content facilitates the deposition of lipophilic compounds like polycyclic aromatic hydrocarbons and some alkylating agents $[42,43]$. Toxic metals like mercury, Iron, and manganese have also been known as risk factors in mitochondria dysfunction as well [44-46]. Another relevant plan of action is awareness of the exposure levels that can cause mitochondrial disruption leading to PD [47]. With this information researchers can develop appropriate therapies to block this disruption. Also, identification of biomarkers complimented by use of genetic mapping and genome-wide association studies (GWAS) would be good preventive counter-measures against Parkinson's disease [48,49]. With the advances of OMICS technologies, it is possible to provide improved risk assessment, monitoring, and prevention planning. Once established, it is possible to use relevant biomarkers to detect cellular malfunctions and apply appropriate treatments. Understanding the underlying cellular mechanisms of PD and its interaction with certain environmental factors will prove advantageous to developing targeted treatments and also, prevent early-onset in young individuals.

\section{Conclusion and Perspective}

Parkinson's disease (PD) is caused by mitochondrial dysfunction in relation to certain defects in proteins, cellular pathways, metabolites, and exposure to pesticides. The primary insult is reduced dopamine levels in the brain due to either biological or environmental factors. There is especially high risk of developing mitochondrial defects and resultant neurodegenerative disease with occupational exposure from pesticides. OMICS techniques have helped with identifying certain biomarkers with possibility of creating medicines that can help with prevention. OMICS has the potential to improve the quality of health care and alleviate the public health burden of disease through more advanced risk assessment and detection. Those afflicted with mitochondrial disorders will serve to benefit from OMICS strategies in development and maximization of targeted treatments. The multiple biochemical and molecular techniques offered by the field of OMICS will prove to be advantageous for the biomedical and public health communities. Overall, the application of OMICS as an integrated molecular assessment tool for diagnostics by exploring biomarkers and disease management of Parkinson's in early onset of pathogenesis can be beneficial in reducing occurrence in susceptible PD population.

\section{References}

1. Kelishadi R (2012) Environmental pollution: Health effects and operational implications for pollutants removal. Journal of Environmental and Public Health 2012: 341637

2. Todorovic M, Newman JR, Shan J, Bentley S, Wood SA, et al. (2015) Comprehensive assessment of genetic sequence variants in the antioxidant 'master regulator' NRF2 in idiopathic Parkinson's disease. PLoS One 10 e0128030.

3. Lesage S, Brice A (2009) Parkinson's disease: from monogenic forms to genetic susceptibility factors. Hum Mol Genet 18: R48-59.

4. Agúndez JA, Jiménez-Jiménez FJ, Luengo A, Molina JA, Ortí-Pareja M, et al. (1998) Slow allotypic variants of the NAT2 gene and susceptibility to earlyonset Parkinson's disease. Neurology 51: 1587-1592.

5. Godeiro C, Aguiar PM, Felício AC, Barsottini OG, Silva SM, et al. (2010) PINK1 polymorphism IVS1-7 A-->G, exposure to environmental risk factors and anticipation of disease onset in Brazilian patients with early-onset Parkinson's Disease. Neurosci Lett 469: 155-158. 
Citation: Kim HD, Warner S, Singh K, Cho JH, Heck DE (2016) OMICS as Therapeutic Platform: Environmental Factors to Parkinson's Disease. J Microb Biochem Technol 8: 222-225. doi: 10.4172/1948-5948.1000289

6. Logroscino $\mathrm{G}$ (2005) The role of early life environmental risk factors in Parkinson disease: What is the evidence? Environ Health Perspect 113: 1234-1238.

7. Chen P, DeWitt MR, Bornhorst J, Soares FA, Mukhopadhyay S, et al. (2015) Age- and manganese-dependent modulation of dopaminergic phenotypes in a C. elegans DJ-1 genetic model of Parkinson's disease. Metallomics 7: 289-298.

8. Kumudini N, Uma A, Naushad SM, Mridula R, Borgohain R, et al. (2014) Sexual dimorphism in xenobiotic genetic variants-mediated risk for Parkinson's disease. Neurol Sci 35: 897-903.

9. Zhou H, Huang C, Tong J, Xia XG (2011) Early exposure to paraquat sensitizes dopaminergic neurons to subsequent silencing of PINK1 gene expression in mice. Int J Biol Sci 7: 1180-1187.

10. Tanner CM, Goldman SM, Aston DA, Ottman R, Ellenberg J, et al. (2002) Smoking and Parkinson's disease in twins. Neurology 58: 581-588.

11. Leung MC, Rooney JP, Ryde IT, Bernal AJ, Bess AS, et al. (2013) Effects of early life exposure to ultraviolet $C$ radiation on mitochondrial DNA content, transcription, ATP production and oxygen consumption in developing Caenorhabditis elegans. BMC Pharmacol Toxicol 14: 9.

12. Bacman SR, Williams SL, Pinto M (2013) Specific elimination of mutan mitochondrial genomes in patient-derived cells by mitoTALENs. Nature Medicine 19: 1111-1113.

13. Schmidt CW (2010) Unraveling environmental effects on mitochondria Environmental Health Perspectives 118: A292-A297.

14. Moorjani N, Westaby S, Narula J, Catarino PA, Brittin R, et al. (2009) Effects of left ventricular volume overload on mitochondrial and death-receptor-mediated apoptotic pathways in the transition to heart failure. Am J Cardiol 103: 12611268.

15. Graf WD, Marin-Garcia J, Gao HG, Pizzo S, Naviaux RK, et al. (2000) Autism associated with the mitochondrial DNA G8363A transfer RNA(Lys) mutation. J Child Neurol 15: 357-361.

16. Perier C, Vila M (2012) Mitochondrial biology and Parkinson's disease. Cold Spring Harb Perspect Med 2: a009332.

17. Hang L, Thundyil J, Lim KL (2015) Mitochondrial dysfunction and Parkinson disease: A Parkin-AMPK alliance in neuroprotection. Ann N Y Acad Sci 1350: $37-47$

18. Morris G, Berk M (2015) The many roads to mitochondrial dysfunction in neuroimmune and neuropsychiatric disorders. BMC Med 13: 68.

19. Bir A, Sen O, Anand S, Khemka VK, Banerjee P, et al. (2014) a-Synucleininduced mitochondrial dysfunction in isolated preparation and intact cells: implications in the pathogenesis of Parkinson's disease. J Neurochem. 131: 868-877.

20. Zanellati MC, Monti V, Barzaghi C, Reale C, Nardocci N, et al. (2015) Mitochondrial dysfunction in Parkinson disease: Evidence in mutant PARK2 fibroblasts. Front Genet 6: 78.

21. Merwe C, Loos B, Swart C, Kinnear C (2014) Mitochondrial impairment observed in fibroblasts from South African Parkinson's disease patients with parkin mutations. Biochem Biophys Res Commun 447: 334-340.

22. Egensperger R, Kösel S, Schnopp NM, Mehraein P, Graeber MB (1997) Association of the mitochondrial tRNA(A4336G) mutation with Alzheimer's and Parkinson's diseases. Neuropathol Appl Neurobiol 23: 315-321.

23. Riemen G, Michaelis G (1993) A point mutation in the core subunit gene of yeast mitochondrial RNA polymerase is suppressed by a high level of specificity factor MTF1. Mol Gen Genet 237: 49-57.

24. Tanner CM, Kamel F, Ross GW, Hoppin JA, Goldman SM, et al. (2011) Rotenone, paraquat and Parkinson's disease. Environ Health Perspect 119: 866-872.

25. Shults CW, Nasirian F, Ward DM, Nakano K, Pay M, et al. (1995) Carbidopa/ levodopa and selegiline do not affect platelet mitochondrial function in early parkinsonism. Neurology 45: 344-348.

26. Segura-Aguilar J, Paris I, Muñoz P, Ferrari E, Zecca L, et al. (2014) Protective and toxic roles of dopamine in Parkinson's disease. J Neurochem 129: 898-915.

27. Müller T, Muhlack S (2012) Cysteine decrease following acute Levodopa intake in patients with Parkinson's disease. Neurosci Lett 521: 37-39.

28. Carta M, Carlsson T, Muñoz A, Kirik D, Björklund A (2008) Serotonin-dopamine interaction in the induction and maintenance of L-DOPA-induced dyskinesias. Prog Brain Res 172: 465-478.

29. Walter $U$ (2009) Transcranial brain sonography findings in Parkinson's disease: Implications for pathogenesis, early diagnosis and therapy. Expert Rev Neurother 9: 835-846.

30. Birkmayer W, Riederer P, Ambrozi L, Youdim MB (1977) Implications of combined treatment with 'Madopar' and L-deprenil in Parkinson's disease. A long-term study. Lancet 1: 439-443.

31. Goodman WK, Charney DS (1985) Therapeutic applications and mechanisms of action of monoamine oxidase inhibitor and heterocyclic antidepressant drugs. J Clin Psychiatry 46: 6-24

32. Elizan TS, Yahr MD, Moros DA, Mendoza MR, Pang S, et al. (1990) L-deprenyl, a MAO-B inhibitor, as an adjunct to conventional L-dopa therapy in Parkinson's disease: Experience in 200 patients. Adv Neurol 53: 431-435.

33. Kalinderi K, Bostantjopoulou S, Fidani L (2016) The genetic background of Parkinson's disease: current progress and future prospects. Acta Neurol Scand

34. Elbaz A, Carcaillon L, Kab S, Moisan F (2016) Epidemiology of Parkinson's disease. Rev Neurol (Paris) 172: 14-26.

35. Bellou V, Belbasis L, Tzoulaki I, Evangelou E, loannidis JP (2016) Environmenta risk factors and Parkinson's disease: An umbrella review of meta-analyses. Parkinsonism Relat Disord 23: 1-9.

36. Marttila RJ, Rinne UK (1981) Epidemiology of Parkinson's disease--an overview. J Neural Transm 51: 135-148.

37. Chiou SM (2015) Sex-related prognostic predictors for Parkinson disease undergoing sub-thalamic stimulation. World Neurosurg 84: 906-912.

38. Barh D, Blum K (2012) OMICS Biomedical perspectives and applications. Boca Raton, FL: CRC Press.

39. Wong C (2013) Biochemical and molecular methods for the study of mitochondrial disorders. Mitochondrial Disorders Caused by Nuclear Genes, Springer, Houston, USA.

40. Human Health Risk Assessment Risk Assessment US EPA Epa.gov.

41. Chen R, Mias G, Li-Pook-Than J, Jiang L, Lam H, et al. (2012) Personal OMICS profiling reveals dynamic molecular and medical phenotypes. Cell 148: 1293-1307.

42. Caudle WM, Bammler TK, Lin Y, Pan S, Zhang J (2010) Using 'OMICS' to define pathogenesis and biomarkers of Parkinson's disease. Expert Rev Neurother 10: 925-942.

43. Chaiswing L, Cole MP, Clair DK, Ittarat W, Szweda LI, et al. (2004) Oxidative damage precedes nitrative damage in adriamycin-induced cardiac mitochondrial injury. Toxicol Pathol 32: 536-547.

44. Meyer JN, Leung MC, Rooney JP, Sendoel A, Hengartner MO, et al. (2013) Mitochondria as a target of environmental toxicants. Toxicol Sci 134: 1-17.

45. Houston MC (2011) Role of mercury toxicity in hypertension, cardiovascular disease and stroke. J Clin Hypertens (Greenwich) 13: 621-627.

46. Shen XM, Dryhurst G (1998) Iron- and manganese-catalyzed autoxidation of dopamine in the presence of L-cysteine: Possible insights into iron- and manganese-mediated dopaminergic neurotoxicity. Chem Res Toxicol 11: 824-837.

47. Monteiro JP, Oliveira PJ, Jurado AS (2013) Mitochondrial membrane lipid remodeling in pathophysiology: A new target for diet and therapeutic interventions. Prog Lipid Res. 52: 513-528.

48. Klein C, Westenberger A (2012) Genetics of Parkinson's disease. Cold Spring Harb Perspect Med 2: a008888.

49. Verstraeten A, Theuns J, Van Broeckhoven C (2015) Progress in unraveling the genetic etiology of Parkinson disease in a genomic era. Trends Genet 31 140-149. 